\title{
La durometría en la valoración de la psoriasis en placas, un estudio exploratorio
}

The durometry in the assessment of psoriasis plate, an exploratory study

\author{
Jhonatan Quintero', Felipe Jaramillo-Ayerbe², Ana María Hoyos³ ${ }^{3}$ José Arnoby Chacón \\ 1. Médico cirujano, residente de tercer año de Dermatología, Universidad de Caldas, Manizales, Colombia \\ 2. Médico cirujano, dermatólogo, dermatopatólogo; jefe, Programa de Dermatología, Universidad de Caldas, Manizales, Colombia \\ 3. Médica cirujana, dermatóloga, Universidad de Caldas, docente, Programa de Dermatología, Universidad de Caldas, Manizales, \\ Colombia \\ 4. Médico, MSP; profesor, Universidad de Caldas, Manizales, Colombia
}

\section{RESUMEN}

Introducción: La psoriasis exhibe cambios biomecánicos en la dureza de la piel; no existen marcadores para medir su actividad y su seguimiento se basa en la 'clinimetría'. Una de las deficiencias del PASI (Psoriasis Area Severity Index) es la interdependencia entre sus variables y la controversia al definir la induración de la placa, según su altura o su semiología. El durómetro, un aparato que mide la dureza de materiales, fue probado por primera vez para medir esta característica. Métodos: Dos evaluadores con un durómetro calibrado de fábrica, midieron en unidades de dureza placas de psoriasis y piel 'sana' como control. Los resultados se analizaron con la t de Student y el ANOVA para grupos pareados con medidas repetidas, el coeficiente de correlación de Pearson y la varianza de Fisher, y la correlación y la concordancia con regresión lineal e índice kappa.

Resultados y conclusiones: Se midieron 33 placas de 13 pacientes con un PASI promedio de 11. Los evaluadores obtuvieron un coeficiente de Pearson de 0,37 para la durometría y de 0,22 para el PASI. La varianza fue mayor de 20 entre las placas y la piel sana. Entre evaluadores, el coeficiente de Pearson fue de 0,95 y el índice kappa de o,88 con un índice de confianza del 95\% y una p menor de 0,05.

La durometría no se correlacionó con el PASI. Sin embargo, es factible una recalibración en su función, mediante más estudios con una muestra de mayor tamaño y analizando los efectos del esquema terapéutico en el tiempo. La durometría es útil en la valoración de la dureza de la placa de psoriasis, con adecuadas correlación y concordancia entre evaluadores.

PALABRAS CLAVE: psoriasis, placa, induración, dureza.

\section{SUMMARY}

Background: Psoriasis biomechanically exhibits changes in the hardness of the skin; there are no markers to measure their activity and their follow-up is based on clinimetry. One of the deficiencies of the PASI is the interdependence between its variables and the controversy when defining the "induration" of the plate, either from its height or from semiology. The durometer, an
Correspondencia:

Jhonatan Quintero

Email:

Jhonaq084@gmail.com

Recibido: $31 / 01 / 18$

Aceptado: 07/05/18

Conflictos de interés:

No se reportan conflictos de interés.

\section{Financiación:}

Ninguna. 
apparatus that measures the hardness of materials, was tested for the first time to measure this characteristic.

Methods: Two evaluators (E1 and E2) with a factory calibrated durometer, measured in hardness units both plaques and "healthy" skin control. The analysis was performed using Student's t-test and ANOVA for paired groups with repeated measures, Pearson's correlation coefficient $(r)$, Fisher's variance $(F)$ and correlation and agreement using linear regression and Kappa index.

Results and conclusions: Thirty-three plaques were obtained from 13 patients with average PASI of 11. E1 and E2 obtained $\mathrm{r}=0.37$ and 0.22 between the durometry vs. IndPASI. An F>20 between plates vs. healthy skin; Inter-rater an $\mathrm{r}=0.95$, and a kappa index of 0.88 with $95 \% \mathrm{CI}$ and $\mathrm{p}<0.05$. Durometry does not correlate with PASI. However, a recalibration in its function is feasible, requiring more studies with a larger sample, analyzing the effects of the therapeutic scheme over time. The durometry is useful in the assessment of the hardness of the psoriasis plaque, with adequate correlation and interrater agreement.

KEY WORDS: Psoriasis, plaque, induration, hardness.

\section{INTRODUCCIÓN}

La psoriasis es una enfermedad inflamatoria crónica de la piel determinada por factores genéticos, inmunológicos y ambientales, y caracterizada por la aparición paralela de hiperproliferación epidérmica, inflamación y angiogénesis ${ }^{(1)}$. Afecta a 125 millones de personas, aproximadamente, en todo el planeta, con una prevalencia promedio del 2 al $3 \%$ de la población mundial. Tiene dos picos de presentación, uno entre los 20 y los 30 años y otro entre los 50 y los 60 años, aunque puede presentarse a cualquier edad. Existe una ligera mayor prevalencia en las mujeres, aunque en los hombres las manifestaciones clínicas son más graves ${ }^{(2-4)}$.

La hipótesis más ampliamente aceptada y reconocida sobre su fisiopatología se fundamenta en que las lesiones dérmicas se originan por la pérdida de la regulación de las interacciones de los componentes innatos y los adaptativos del sistema inmunológico con las células residentes de la piel, que lleva a una epidermopoyesis acelerada como reacción a la inflamación por activación del linfocito $\mathrm{T}$ y sus citocinas inflamatorias. Si hay progresión de la enfermedad, se asocia con inflamación sistémica y enfermedades con- comitantes como la artritis psoriásica, la enfermedad cardiovascular, la diabetes y la depresión. En el 2014, la Organización Mundial de la Salud (OMS) la declaró un problema global de salud, acorde con el reporte de carga de la enfermedad, la cual se equipara con la de enfermedades tan graves como el infarto agudo del miocardio $^{(1,3-5)}$.

La psoriasis posee múltiples presentaciones clínicas, de las cuales la más característica y frecuente es la psoriasis vulgar (80 a $90 \%$ ), seguida por la guttata o en gotas (10\%) y la pustulosa (menos del $5 \%$ ); otras menos frecuentes son la ungular que puede asociarse con la vulgar, la eritrodérmica y la inversa. Existen otras formas raras, como la fotosensible, la folicular, la espinosa la congénita eritrodérmica, la verrugosa anular, la de tipo eritema gyratum repens y la lineal o nevoide ${ }^{(2,4-6)}$.

En Colombia, los datos epidemiológicos de la enfermedad son escasos y solo se dispone de reportes de las tres principales ciudades del país. Entre estos, el que más se asemeja en sus resultados a los de la literatura internacional es el de González, et al. ${ }^{(7)}$, llevado a cabo en el Hospital Militar Central de Bogotá entre julio de 2007 y julio de 2008. Se encontró que la enfermedad se había iniciado, en la mayoría de los casos, después de los 50 años y la psoriasis vulgar fue la más frecuente (89\%), seguida por la guttata (7\%); además, hubo dos casos de psoriasis eritrodérmica, uno de psoriasis inversa y otro de psoriasis pustulosa. En otro estudio, se determinó una frecuencia de $3 \%$ en toda la consulta dermatológica de un centro de referencia ${ }^{(4,7)}$.

A pesar de que la psoriasis vulgar es la más frecuente, no hay biomarcadores establecidos para medir su actividad. De manera experimental, se ha intentado estudiar físicamente la placa de psoriasis mediante el ultrasonido $(20 \mathrm{mHz})$, la colorimetría y los estudios de imágenes ${ }^{(8)}$; también, se ha analizado mediante "la estratificación histológica del calibre (gruesa y delgada) determinando sus fenotipos moleculares e implicaciones clínicas” ${ }^{5}$. Sin embargo, estos son métodos poco prácticos, algunos costosos, dependen del examinador y la posibilidad de usarlos en la práctica diaria es limitada. Por consiguiente, el seguimiento de la enfermedad se basa actualmente en la evaluación clínica por individuos entrenados en el uso de diversas herramientas, en su mayoría conformadas por escalas 'clinimétricas' de medición, para evaluar su gravedad y pronóstico en un momento dado ${ }^{(9)}$.

Entre estas herramientas, el PASI (Psoriasis Area Severity Index), descrito en 1978 por Fredrikson y Petterson, es una medida que se limita a valorar la placa psoriásica y su extensión en la superficie corporal. Ac- 
tualmente, la Food and Drug Administration (FDA) lo considera un parámetro de la eficacia de los productos biológicos en los estudios clínicos y se ha convertido en la escala más usada, principalmente el PASI $_{75}$ (mejoría del $75 \%$ del PASI basal) ${ }^{(4,10)}$.

No obstante, existen problemas asociados con el PASI, como que carece de sensibilidad cuando el área corporal afectada es de menos del $10 \%$, y que las tres características de la placa (eritema, descamación e induración) son interdependientes, semicuantitativas y, por ende, su calificación está sujeta a la experiencia y la subjetividad del examinador; por esta razón, puede considerarse un índice poco práctico y de discutible precisión, principalmente, cuando lo determina un examinador inexperto ${ }^{3-5,10)}$. Además, existe controversia sobre la determinación de las características de la placa, como su altura y tamaño. En internet, se encuentran algunas calculadoras disponibles para el PASI (como el PASI Training ${ }^{\mathrm{TM}}$ 2008) ${ }^{(11)}$, que califica de leve, una altura de $0,25 \mathrm{~mm}$, de moderada, una de $0,5 \mathrm{~mm}$, de grave, una de $1 \mathrm{~mm}$, y de muy grave, una de 1,25 mm. En cuanto a su gravedad, una induración pequeña se considera leve, una fácilmente palpable y de escasa altura, moderada, una definitivamente elevada con induración fácilmente palpable, grave, y una elevada con importante induración o 'liquenificación', muy grave ${ }^{(12)}$, lo cual genera confusión al momento calificar este aspecto en particular.

Los durómetros, desarrollados por Albert Shore en la década de 1920, son dispositivos de mano "que miden por un sistema de resortes, la dureza de un material y están validados internacionalmente como instrumentos industriales utilizados para medir la dureza del caucho, del plástico y otros materiales no metálicos” (13-15).

Desde la década de 1970 y hasta la fecha, en la literatura médica se vienen usando los durómetros en varios campos como en neurocirugía, para valorar la presión intracraneal ${ }^{(16)}$; en cirugía cardiovascular, para medir la dureza del miocardio ${ }^{(17)}$, y en cirugía general, para evaluar la dureza pancreática ${ }^{(18)}$. También se ha usado para valorar la enfermedad venosa, principalmente en la lipodermatoesclerosis, con óptimos resultados ${ }^{(14,19)}$. Además, se ha logrado comprobar su validez, fiabilidad y reproducibilidad en la medición de la induración de la piel de adultos y niños en la esclerosis localizada y en la sistémica.

En las enfermedades que se manifiestan clínicamente por cambios en la dureza cutánea, principalmente la esclerosis, la ‘durometría' se ha descrito como una herramienta no invasiva, económica, portátil y práctica. La puede usar adecuadamente el personal no entre- nado, aprendiendo a utilizarse tan solo en un promedio de 10 a 15 minutos; además, su buena precisión por parte de los evaluadores y entre evaluadores y su óptima correlación con las medidas clinimétricas, invitan a estudiar su desempeño en otras entidades ${ }^{(13,15}$, 20-22).

Teniendo en cuenta lo anterior, el propósito de este estudio fue analizar una de las tres características físicas que siempre han permanecido dependientes al valorar la psorasis vulgar con el PASI, usando el durómetro como instrumento autónomo para medir la induración como una variable cuantitativa objetiva e individual, que permite analizar sus propiedades métricas.

Hasta donde sabemos, no existe un estudio similar sobre la psoriasis vulgar, el cual se facilita por la relativa frecuencia de esta enfermedad en la consulta dermatológica.

Para su desarrollo, el objetivo general fue establecer la posibilidad de usar la ‘durometría' para medir objetivamente la induración de la placa psoriásica; y los objetivos específicos fueron correlacionar la 'durometría' con la induración determinada mediante el PASI y analizar la concordancia entre observadores.

\section{PACIENTES Y MÉTODOS}

Se llevó a cabo un estudio para evaluar la 'durometría', mediante muestreo no probabilístico y por conveniencia, en los pacientes con psoriasis vulgar que asistieron a consulta dermatológica entre marzo de 2016 y febrero de 2017, dentro del marco del convenio de docencia del programa de especialización de la Universidad de Caldas en Manizales (Colombia).

Se incluyeron los pacientes con lesiones de psoriasis vulgar en el tronco, los miembros superiores o los inferiores, que no estuvieran localizadas en las prominencias óseas. Se excluyeron aquellos con lesiones localizadas en áreas correspondientes a prominencias óseas, como el cuero cabelludo, los codos, las rodillas o los tobillos, entre otras.

Todos los pacientes incluidos en el estudio firmaron el correspondiente consentimiento informado, teniendo en cuenta la Declaración de Helsinki de 1964 y la Resolución 8430 de 1993 del Ministerio de Salud. El estudio fue aprobado mediante el Acta No 006 de 2016 de los Comités de Ética y Bioética en Investigación de la Facultad de Ciencias para la Salud de la Universidad de Caldas.

Una dermatóloga experta determinó el PASI en la consulta. La dureza de la placa se midió con un durómetro análogo (Rex Tipo Calibre oo ${ }^{\mathrm{TM}}$; Rex Gauge, Buffalo 
Grove, IL, USA, con calibración de fábrica) con un rango de o a 100 unidades de dureza establecidas arbitrariamente por el fabricante. El modelo oo está diseñado para medir la dureza de tejido textil y animal. En su extremo distal, consta de un botón penetrante (indenter tip) que, al entrar en contacto por gravedad con la superficie valorada, activa un sistema de resortes conectado a un reloj de 0,5 unidades de dureza de precisión (figura 1).

Originalmente, las mediciones se graduaron para correlacionarlas con la induración según el PASI, así: induración baja, de o a 25 unidades de dureza; intermedia, de 26 a 50; alta, de 51 a 75, y de muy alta dureza, de 76 a 100.

No obstante, después del análisis de las placas, estos rangos se recodificaron en función de la menor y la mayor dureza representadas en la base de datos, con lo cual los grupos quedaron así: baja, menos de 22 unidades de dureza; intermedia, de 23 a 29; alta, de 30 a 32, y muy alta, de más de 33, para el manejo de los datos.

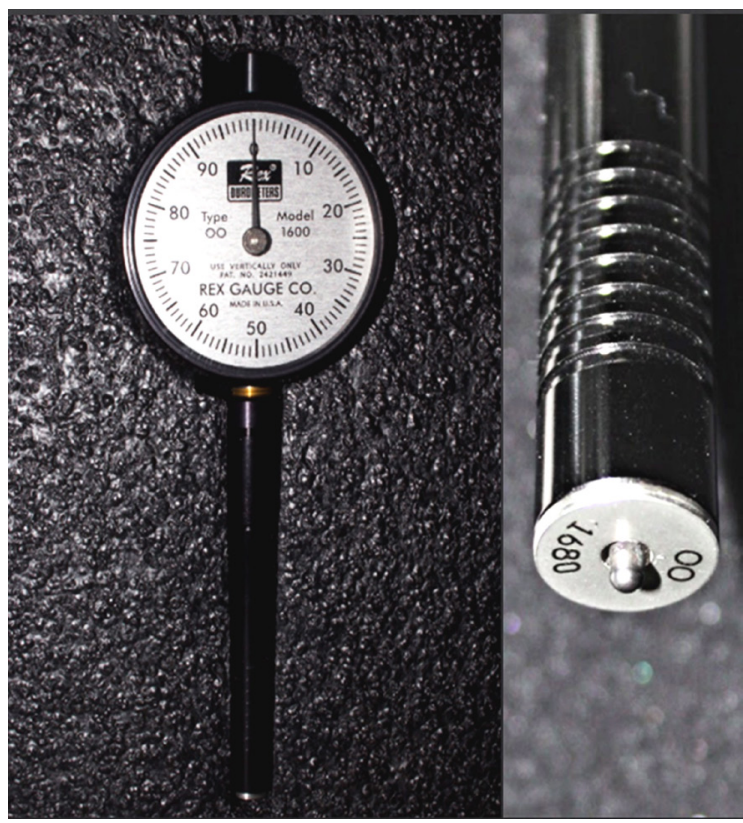

Figura 1. Durómetro REX GAUGE ${ }^{\mathrm{TM}}$, modelo oo.
Estas mediciones se obtuvieron en una sola consulta clínica. El durómetro se colocaba perpendicular a la lesión en la zona de mayor grosor -previamente demarcada por mutuo acuerdo entre los dos evaluadores-, dejándolo reposar por gravedad sobre la placa, y manteniendo relajados los músculos de la zona en estudio mediante un adecuado apoyo. En el tronco, la medida se obtuvo siempre durante la espiración (figura 2). Cada evaluador registraba el dato después de 10 a 15 segundos de contacto del aparato con la placa.

Se hicieron tres mediciones en cada lesión para evitar un error accidental. La media de las mediciones se utilizó como dato ‘duro’ para el análisis estadístico. Como control, se tomaron medidas en piel clínicamente sana de un área adyacente o contralateral a la zona anatómica afectada.

El análisis de los datos se hizo mediante la prueba t de Student y el ANOVA para grupos pareados con medidas repetidas, el coeficiente de correlación de Pearson y la varianza de Fisher.

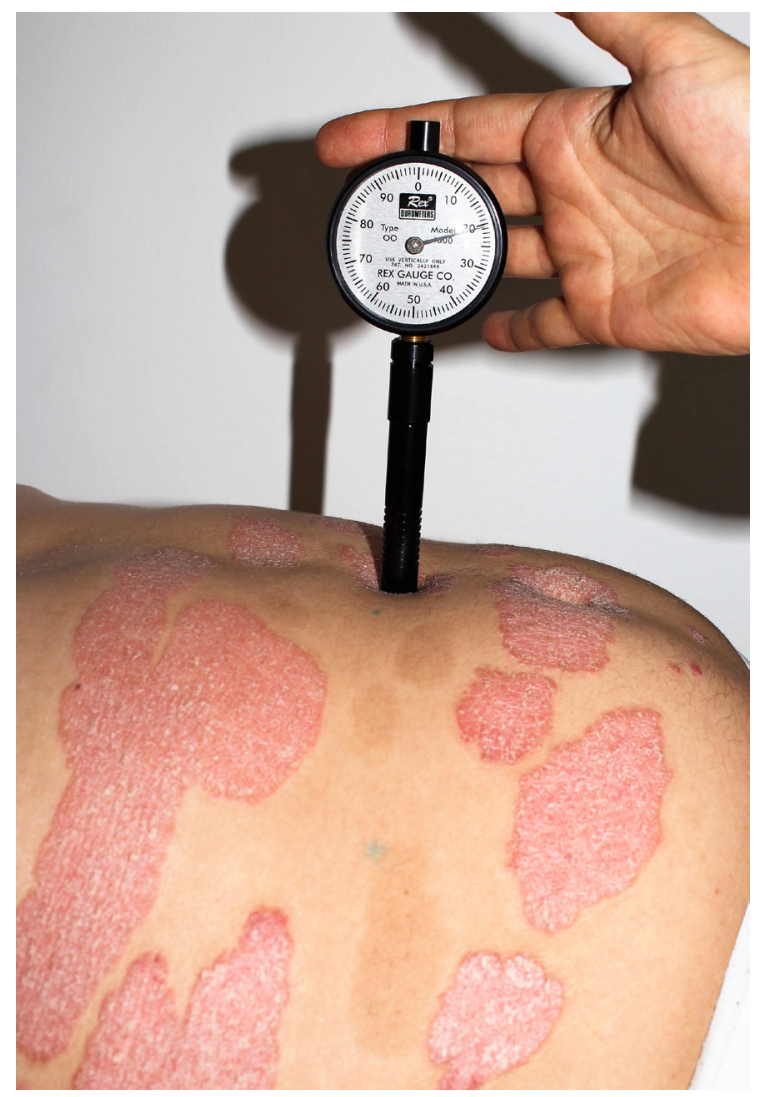

Figura 2. El durómetro se coloca perpendicular a la lesión, dejándolo reposar por gravedad sobre la placa, mientras que la zona anatómica afectada evaluada se apoya para mantener los músculos subyacentes relajados. En el tronco, la dureza se valoró durante la fase espiratoria de la respiración. 
Se realizó regresión lineal de los resultados de la 'durometría’ categorizada en cuartiles (1 a 4), con la induración aportada por el PASI considerada como parámetro de referencia. Esta medida se calculó según el método semiológico indicado por Trujillo, et al., en su glosario para ensayos clínicos en psoriasis, el cual categoriza la induración de la placa de 1 a 4, así: leve, cuando es una pequeña induración; moderada, cuando es fácilmente palpable, con escaso aumento del nivel de la piel; grave, cuando está evidentemente elevada, con induración fácilmente palpable; y muy grave, cuando es una lesión elevada y con induración grave o 'liquenificación' ${ }^{(13)}$.

También, se analizaron la correlación y la concordancia de las 'durometrías' entre observadores, mediante regresión lineal y el índice kappa de Cohen con ponderación por pesos cuadráticos ${ }^{(23)}$. Los datos secundarios se analizaron por distribución de frecuencias y se consideró un valor de p menor de 0,05 para la significación estadística. La edad, el sexo, la región anatómica de la placa evaluada y el fototipo según la clasificación de Fitzpatrick, se analizaron como datos secundarios por distribución de frecuencias.

Los datos estadísticos se procesaron con el software Epi-Info 3.5 y EpiData entry, versión 2.0.

\section{RESULTADOS}

\section{Características epidemiológicas y gravedad de la enfermedad}

Se evaluaron 13 pacientes con 33 placas, 10 hombres (77 \%) y 3 mujeres ( $23 \%$ ), $7,7 \%$ con piel de fototipo Fitzpatrick II, 76,9 \% con fototipo III y $15,4 \%$ con fototipo IV. El rango de edad fue de 16 a 79 años, con una mediana de 53.

La media del PASI determinado por el experto fue de 11, con un rango de 1,8 a 37,9. Según la induración como parámetro de referencia, se puntuaron: 8 (24,2 \%) placas con induración leve, 13 (39,4\%), con induración moderada, 9 (27,3 \%) con induración grave, у 3 (9,1 $\%)$ con induración muy grave (tabla 1).

\section{Datos 'duros' analizados, correlación y concordancia}

Teniendo en cuenta que se debían obtener tres medidas consecutivas, se practicaron 198 'durometrías' sobre las placas de psoriasis, y 198 mediciones sobre la piel sana contralateral, entre ambos evaluadores.

Tabla 1. Características clínico-epidemiológicas de los pacientes con psoriasis vulgar y PASI de la induración de la placa de psoriasis como unidad de análisis

$\begin{array}{lcc} & n & \% \\ \text { Edad } & & 100 \\ 16 \text { a } 79 \text { años } & 13 & \\ \text { Media: } 48,3 & & 23 \\ \text { Sexo } & 3 & 77 \\ \text { Femenino } & 10 & \\ \text { Masculino } & & 7,7 \\ \text { Fototipo } & & 76,9 \\ \text { I } & 1 & 15,4 \\ \text { II } & 10 & \\ \text { III } & 2 & \\ \text { IV } & & \\ \text { V } & & 7,7 \\ \text { VI } & & 61,5 \\ \text { PASI } & 1 & 30,8 \\ \text { Leve } & 8 & 100 \\ \text { Moderado } & 4 & 24,2 \\ \text { Grave } & 43,3 \\ \text { Induración de la placa } & 33 & 39,4 \\ \text { Leve } & 8 & 9,1 \\ \text { Moderada } & 13 & \\ \text { Grave } & 9 & \\ \text { Muy grave } & 3 & \\ \end{array}$


El ANOVA para más de dos medidas repetidas y paralelas, comparando las 99 medidas de la piel sana, arrojó una varianza menor de $1 \mathrm{y}$ una $\mathrm{p}$ mayor de 0,05 para cada evaluador; la misma tendencia se observó en las 99 medidas de las placas, lo cual indica una baja varianza en las mediciones repetidas en la piel sana y la enferma. Cuando se sometieron a valoración las tres medidas de las placas frente a las tres de su contraparte sana, se obtuvo una varianza mayor de 20 para cada evaluador y una p menor de 0,05, determinando una varianza estadísticamente significativa en la comparación de la piel sana con la enferma (figura 3).

De manera global, el evaluador E1 obtuvo un promedio de 28,34 unidades de dureza con un rango de 8,6 a 56, y una desviación estándar (DE) de 8,9; mientras que el E2 obtuvo un promedio de 27,73 unidades de dureza con un rango de 7,5 a 55,33, y una DE de 9,4. No hubo una diferencia estadísticamente significativa $(p=0,91)$; el coeficiente de correlación de Pearson fue de 0,99, y hubo paridad efectiva entre ambos evaluadores en la evaluación de la piel con placas de psoriasis y la piel sana de control $(\mathrm{p}=0,03)$. Con respecto a la induración de todas las placas aportada por el PASI en comparación con las 'durometrías' de ambos evaluadores, se encontró un coeficiente de correlación de 0,37 y de 0,22

\section{(figuras 4 y 5).}

En el tronco, se valoraron 13 placas. Para el evaluador E1, la media de las 'durometrías' en las placas fue de 25 unidades de dureza con DE de 8,9, y, en la piel sana contralateral, fue de 15,1 unidades de dureza, con una DE de 6,1. Para el E2, el promedio de las 'durometrías' en las placas fue de 23,7 unidades de dureza, con una DE de 8,1 y, en la piel sana de control, fue de 13,7 unidades de dureza con una DE de 5,8 (p<0,05). Hubo paridad entre la 'durometría' y la induración puntuada mediante el PASI en 4 (31 \%) placas según el evaluador E1 y en 5 (38,5 \%) placas según el evaluador E2.

En los miembros superiores, se valoraron seis placas. En ellas, el evaluador E1 obtuvo una media de 32,77 unidades de dureza $(\mathrm{DE}=7,3) \mathrm{y}$, el E2, una media de 34,28 unidades de dureza $(\mathrm{DE}=5,5)$. Sobre la piel sana de control, el E1 obtuvo una media de 25.5 unidades de dureza ( $\mathrm{DE}=7,3)$, y el E2, una media de 24,5 unidades de dureza $(\mathrm{DE}=4,5)$. Hubo paridad entre la 'durometría' y la induración puntuada mediante el PASI en 3 (31\%) placas, según ambos evaluadores.

En los miembros inferiores, se valoraron 14 placas. En ellas, el evaluador E1 obtuvo una media de 29,5 unidades de dureza $(\mathrm{DE}=9,6) \mathrm{y}$, el E2, una media de 29 unidades de dureza $(\mathrm{DE}=10,3)$. En la piel sana de control,

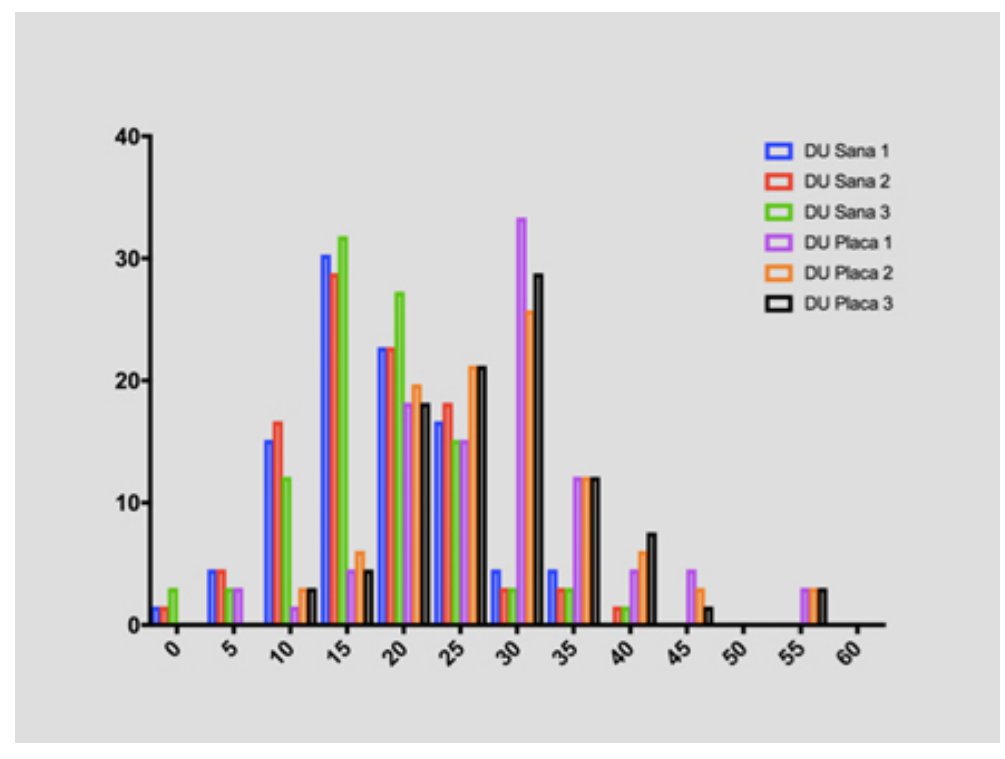

Figura 3. Histograma de medidas repetidas en piel sana y con psoriasis vulgar. ANOVA: nótese la baja varianza (eje de las ordenadas: porcentaje de frecuencia relativa) entre las tres medidas de la 'durometría' (DU eje de las abscisas) en ambos casos. 


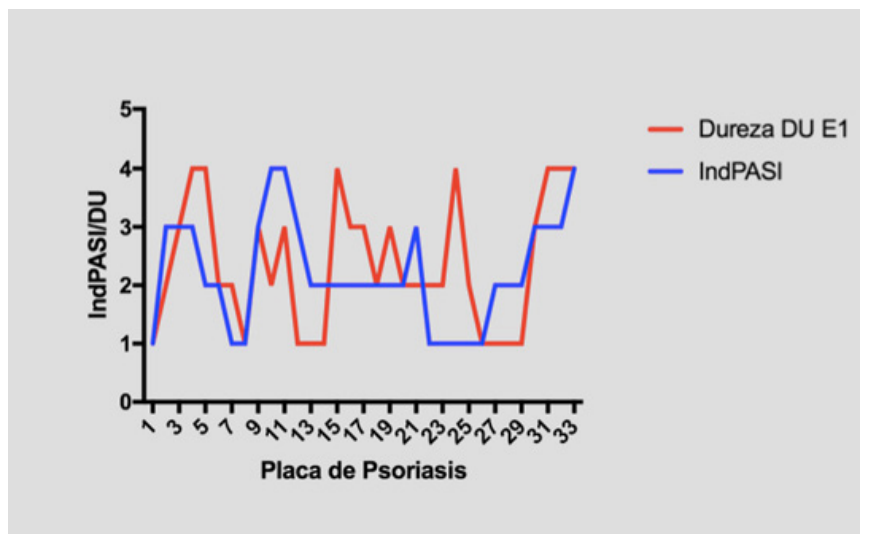

Figura 4. Paridad del $37 \%$ entre la induración aportada por el PASI (IndPASI) y las 'durometrías' del evaluador 1 (E1)

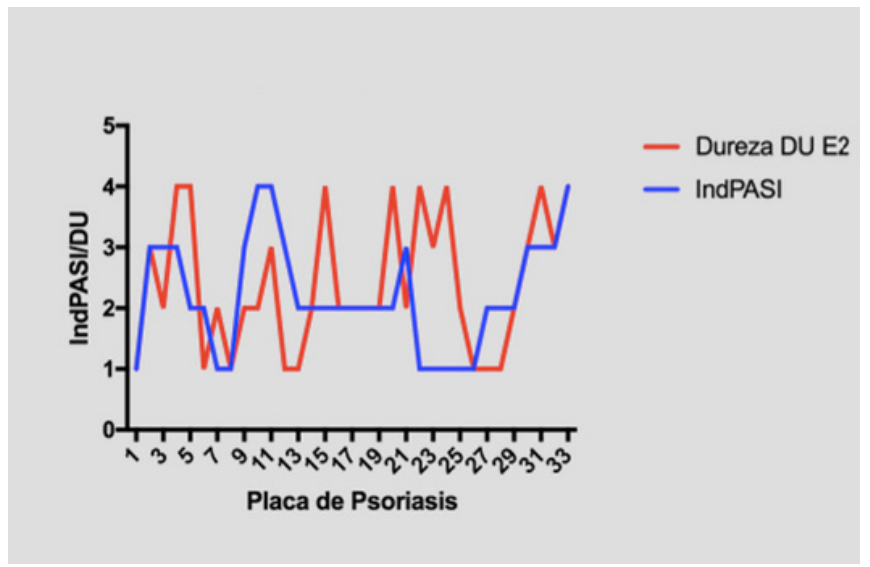

Figura 5. Paridad del $22 \%$ entre la induración aportada por el PASI (IndPASI) y las 'durometrías' del evaluador 2 (E2)

el evaluador E1 obtuvo una media de 19 unidades de dureza $(D E=6,9)$, y el E2, una media de 18 unidades de dureza $(\mathrm{DE}=7,2)(\mathrm{p}<0,05)$. Hubo paridad entre la 'durometría’ y la induración puntuada por el PASI, en 5 (35,7 \%) placas según el evaluador E1 y en 4 (28,6 \%) según el E2 (tabla 2).

De acuerdo con las cuatro posibles categorías de dureza (baja, intermedia, alta y muy alta) y después de la recodificación de los rangos, se compararon los valores obtenidos por el evaluador E1 y por el E2. La dureza fue baja para el E1 en $9(27,25 \%)$ placas y, para el E2, en 8 $(24,25 \%)$; fue intermedia para el E1 en $8(24,25) \mathrm{y}$, para el E2, en 13 (39,25\%); fue alta para el E1 en 7 (21,25\%) y, para el E2, en 4 (12,25\%); y fue muy alta para el E1 en 9 $(27,25 \%)$ y, para el E2, en 8 (24\%).

La prueba t de Student mostró paridad entre las 'durometrías' de ambos evaluadores, tanto para las placas como para la piel sana $(\mathrm{p}<0,05)$. Igualmente, mostró una diferencia estadísticamente significativa de los re- sultados promedio obtenidos por ambos evaluadores en las 'durometrías' de las placas en comparación con las de la piel sana de control, con un valor de $\mathrm{p}<0,05$ y un Intervalo de confianza del $95 \%$. El evaluador E1 obtuvo en promedio una diferencia de 9 unidades de dureza entre las placas y la piel sana de control (rango de 13,44 a 4,89) y, el E2, una de 10 unidades de dureza (rango de 11,87 a 8,64) (figura 6).

Por regresión lineal, se encontró un coeficiente de correlación $\mathrm{r}$ de 0,95 y uno de determinación $\mathrm{r}^{2}$ de 0,91, con unos límites de confianza entre o,82 y 0,95.

Finalmente, la concordancia entre los dos evaluadores se obtuvo calculando el índice kappa, el cual fue igual a 0,88 , con un acuerdo previo esperado de $0,7 \mathrm{y}$ uno observado de 0,97 , con intervalo de confianza de $95 \%$ ( $\mathrm{p}<0,001)$ (tabla 3).

Ningún paciente refirió incomodidad por el uso del durómetro. 
Tabla 2. Paridad entre la dureza según la 'durometría' (DU) y según el PASI (Psoriasis Area Severity Index), por área anatómica evaluada; promedios de las 'durometrías’ con sus desviaciones estándar (DE) para cada evaluador (E1 y E2 ) y su significación estadística ( $\mathrm{p}<0.05)$ al comparar con la piel control o "sana”.

\begin{tabular}{|c|c|c|c|c|c|c|}
\hline Localización & \multicolumn{2}{|c|}{$\mathrm{PASI}=\mathrm{DU}$} & $\begin{array}{l}\text { Media DU Piel } \\
\text { sana }\end{array}$ & $\begin{array}{l}\text { Media DU } \\
\text { Placas }\end{array}$ & $\begin{array}{l}\text { Media DU } \\
\text { Placas }\end{array}$ & \\
\hline $\mathrm{N}=33$ placas & \multicolumn{2}{|c|}{$E_{1} / E_{2}(1-4)$} & $\mathrm{E}_{1} / \mathrm{E}_{2}$ & $\mathrm{E}_{1} \pm \mathrm{DE}$ & $\mathrm{E} 2 \pm \mathrm{DE}$ & $p<0,05$ \\
\hline Tronco & 1) 3 & $(2 / 2)$ & & & & \\
\hline \multirow[t]{3}{*}{13 placas } & 2) 7 & $(1 / 3)$ & & & & \\
\hline & 3) 3 & $(1 / 0)$ & $15,1 / 13,7$ & $25 \pm 8,9$ & $23,7 \pm 8,1$ & Sí \\
\hline & 4) 0 & $(0 / 0)$ & & & & \\
\hline PASI/DU & \multicolumn{2}{|c|}{$31-38,5 \%$} & & & & No \\
\hline Miembro superior & 1) 2 & (o/o) & & & & \\
\hline \multirow[t]{3}{*}{6 placas } & 2) 0 & $(0 / 0)$ & $25,5 / 24,5$ & $32,7 \pm 7,3$ & $34,7 \pm 7,5$ & Sí \\
\hline & 3) 4 & $(3 / 3)$ & & & & \\
\hline & 4) 0 & (o/o) & & & & \\
\hline PASI/DU & \multicolumn{2}{|c|}{$50 \%$} & & & & No \\
\hline Miembro inferior & 1) 3 & $(1 / 1)$ & & & & \\
\hline \multirow[t]{3}{*}{14 placas } & 2) 6 & $(2 / 1)$ & $19 / 18$ & $29,5 \pm 9,6$ & $29 \pm 10,6$ & Sí \\
\hline & 3) 2 & $(1 / 1)$ & & & & \\
\hline & 4) 3 & $(1 / 1)$ & & & & \\
\hline PASI/DU & \multicolumn{2}{|c|}{$28,6-35,7 \%$} & & & & No \\
\hline
\end{tabular}

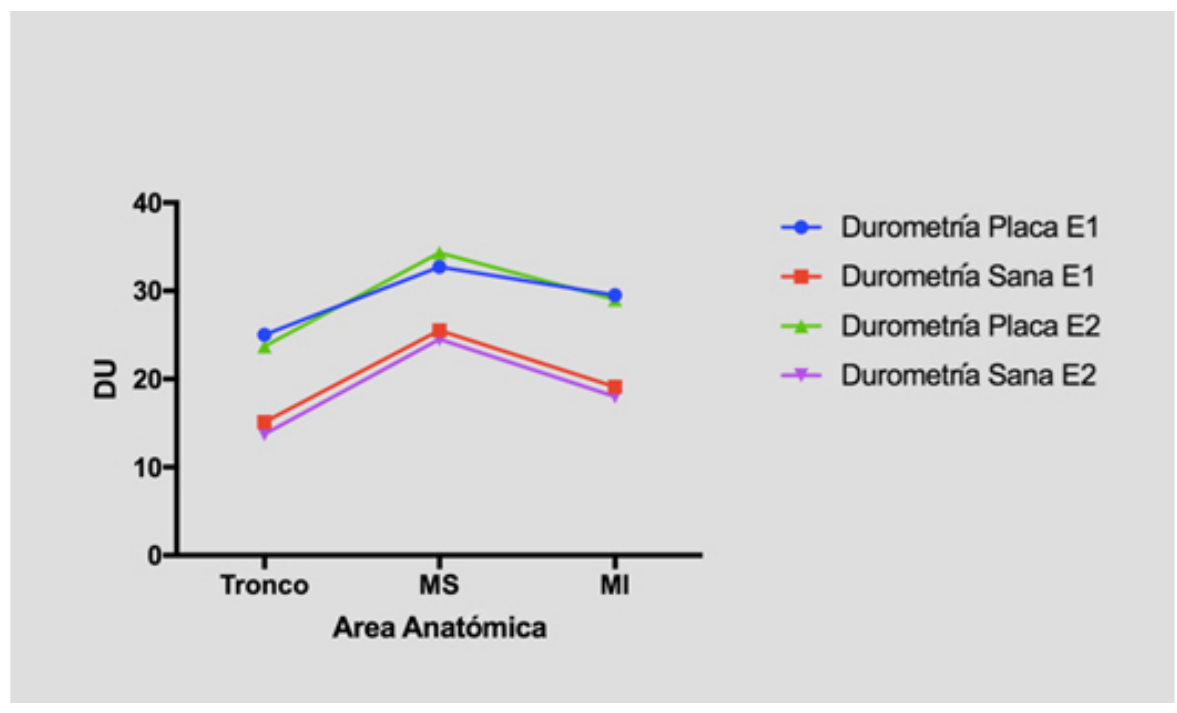

Figura 6. Promedio de la 'durometría' de las placas de psoriasis y de la piel sana: paridad entre las medidas de ambos evaluadores (E1 y E2), con una diferencia promedio entre la piel sana y la enferma de 9 y 10 unidades de dureza, respectivamente 
Tabla 3. Tabla de dispersión: correlación lineal positiva entre las 'durometrías' promedio del evaluador 1 (inexperto) (PROE1) y las 'durometrías' promedio del evaluador 2 (experto) (PROE2)

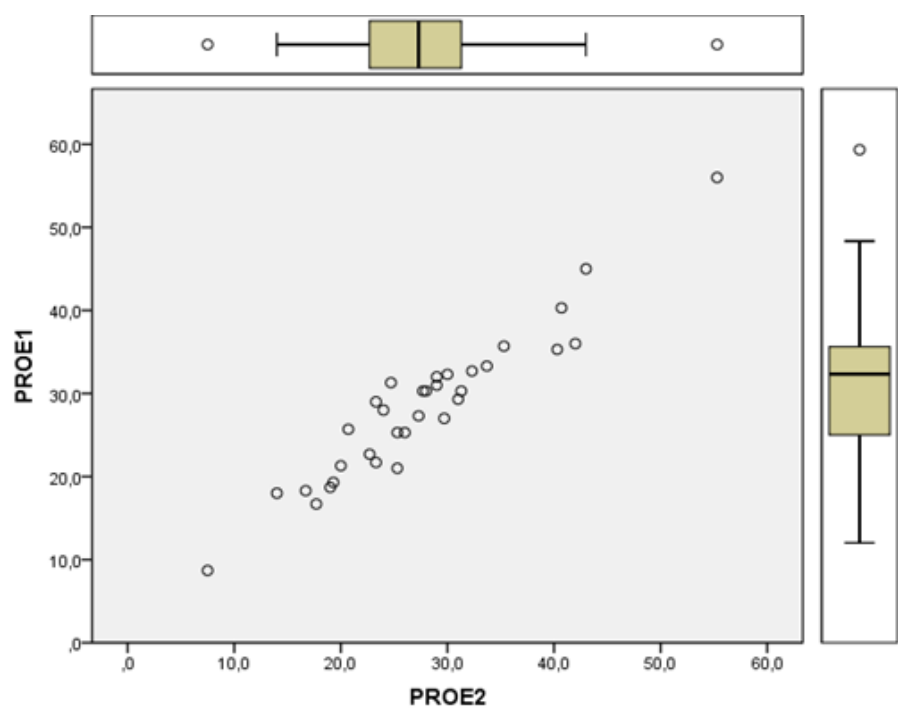

\section{DISCUSIÓN}

La dureza, como componente biomecánico de la piel, puede verse alterada por cambios en el grosor, la densidad o la elasticidad, así como por la presencia de edema tisular ${ }^{(15,20,22,24)}$. En la psoriasis vulgar, esta alteración resulta de la hiperproliferación epidérmica, la vasodilatación y el edema en la dermis papilar ${ }^{(1,2)}$.

En la evaluación clínica y principalmente investigativa, es necesaria una caracterización rutinaria con las diferentes escalas 'clinimétricas' de gravedad, las cuales, por su naturaleza de medición semicuantitativa, son muy propensas al sesgo por subvaloración o sobrevaloración; esto ocurre, principalmente, en manos de evaluadores inexpertos, lo que conlleva errores de clasificación, y expone a los pacientes a tratamientos farmacológicos sistémicos más agresivos y, por ende, a sus efectos secundarios o, por el contrario, dejar a merced de la enfermedad a un enfermo solo con un medicamento tópico, cuando en realidad requería un abordaje sistémico mediante inmunomoduladores convencionales o productos biológicos ${ }^{(25)}$.

Actualmente, múltiples investigaciones se vienen abriendo camino para mejorar el rendimiento del médico tratante al clasificar la gravedad de la psoriasis vulgar en asociación con la 'clinimetría'.

En la literatura disponible revisada, se encontraron métodos invasivos y no invasivos. Entre los invasivos, hay estudios basados en biomarcadores séricos, en los cuales se ha demostrado, por ejemplo, la correlación de niveles séricos elevados de interleucina $16^{(26)}$, el factor de crecimiento 23 derivado de fibroblastos y el factor de crecimiento placentario ${ }^{(27)}$, con formas más graves de la enfermedad; también, se encontraron el seguimiento histopatológico y la determinación fenotípica de la enfermedad por medio de la inmunohistoquímica (CD4, CD8 y CD56), entre otros ${ }^{(28)}$.

Entre los métodos no invasivos, se encontraron estudios de imágenes, como la videocapilaroscopia y la ultrasonografía de alta resolución (20 MHz), y su correlación con la respuesta al tratamiento sistémico con ciclosporina ${ }^{(29)}$, la tipificación de la microcirculación de la placa con respecto a la piel normal por medio de tomografía de coherencia óptica sin medio de contraste ${ }^{(30)}$, y sistemas de apoyo diagnóstico asistidos por computadora mediante redes neurales, máquinas de soporte vectorial o aprendizaje automático, intentando valorar mediante parámetros la gravedad de la psoriasis vulgar en función del PASI, por medio de la segmentación y el análisis de cada uno de sus componentes mediante fotografías estandarizadas ${ }^{(31)}$.

También se encontraron estudios más afines con el presente que miden directamente las propiedades biomecánicas de la placa, como el de Dobrevy, y el de Choi, et 
al. En ellos se utilizaron plataformas multiadaptables de bioingeniería alemana, más reconocidos como "sistemas MPA" (adaptador de múltiples sondas), como el "mexámetro", el "corneómetro" y el "cutómetro". El "mexámetro" mide el color de la piel disparando tres longitudes de onda; mediante el cálculo de la reflexión de la luz, se analiza el color con base en la cantidad de melanina y de hemoglobina de la piel, para analizar el eritema. El "corneómetro" mide el estado de hidratación de la piel, para analizar la descamación. El "cutómetro" o dispositivo de succión no invasivo, usa un sistema de succión y luz óptica conectados a un receptor para determinar la firmeza y la elasticidad de la placa (induración).

Se ha encontrado coherencia y reproducibilidad de los resultados, incluyendo una varianza significativa durante el tratamiento, principalmente con mayor sensibilidad y de manera más temprana en cuanto a la

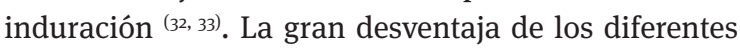
métodos aquí referidos, es que requieren equipo e insumos especializados, personal y tiempo adicionales, lo cual implica una considerable inversión de recursos. Esta falta de precisión y la limitada tecnología disponible en nuestro medio aplicable a la psoriasis vulgar, nos enfrentan al reto de considerar nuevos instrumentos o elementos que, de forma cuantitativa, objetiva, práctica y económica, nos permitan medir la gravedad de este tipo de enfermedades crónicas de alto impacto en salud. El común denominador de afecciones como la psoriasis es la gran carga de morbilidad que no se acompaña de mortalidad directa en su historia natural. Esto se traduce en una marca vitalicia en el historial clínico de los afectados, y genera impactos directos en su calidad de vida, social y económica. Los enfermos pasan a engrosar las filas de los múltiples estudios de farmacoeconomía, principalmente cuando se decide iniciar algún tratamiento con productos biológicos que, como bien sabemos, acarrean un gasto importante para el sistema de salud ${ }^{(25,34,35)}$.

En estudios previos, principalmente sobre esclerosis sistémica y localizada, se obtuvo una correlación positiva estadísticamente significativa entre la 'durometría' y la escala 'clinimétrica' de gravedad MRSS (Modified Rodnan Skin Score), menos compleja que el PASI pero que comparte sus mismas desventajas por ser semi-

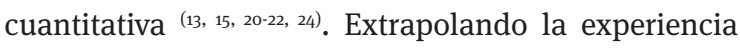
obtenida en ellos, se analizó por primera vez el desempeño de un durómetro en la psoriasis vulgar, usando la piel 'clínicamente sana' como control, y se comparó con el del método estándar de referencia de la 'clinimetría', el PASI.

Se destaca que la mayoría ( $77 \%$ ) de los pacientes en este estudio fueron de sexo masculino que, según los datos epidemiológicos revisados, presentan mayores índices de gravedad (PASI promedio de 11). En la muestra en estudio, predominaron los pacientes mestizos con piel de fototipo III y IV (93,3\%), muy similar a lo informado en la región hasta ahora $\left.{ }^{3}, 4,7\right)$.

En los análisis iniciales de los resultados globales, el PASI y la 'durometría' parecen concordar en $94 \%$ en el número de placas clasificadas como de dureza baja, intermedia o alta; no obstante, al subclasificar por regiones anatómicas y al calcular la regresión lineal de cada placa, se encontró que solamente hay correlación lineal positiva promedio del $22 \mathrm{al} 37 \%$, respectivamente para E1 y E2, entre estos dos instrumentos. Es probable que esto se deba a la interdependencia de las distintas variables físicas analizadas y a su ponderación semiológica o semicuantitativa. Además, el durómetro solo cuantifica la dureza física de la placa, mas no el grado de infiltración también incluido en su análisis.

Los resultados obtenidos mediante regresión lineal y el índice kappa, hablan de una adecuada estabilidad del instrumento con respecto a la medida de la dureza de la placa. Esto se explica por una mínima variación entre evaluadores, que resulta en una medición que, con razonable certeza, puede calificarse como adecuada, proveniente de un instrumento con buena calidad en su precisión y estabilidad métrica.

Se resaltan la nobleza y la simplicidad del durómetro análogo como instrumento para medir la dureza de la placa psoriásica. Mediante unas sencillas y breves directrices técnicas, permite obtener una medida cuantitativa de la dureza y puede ayudar, con cierta seguridad, a diferenciar entre la piel 'sana' contralateral y la lesión examinada.

Se reconocen debilidades en este estudio. En primer lugar, está el indeseado y limitado tamaño de la muestra, ya que pudo interferir al estrecharse los rangos entre alta y muy alta dureza en la recodificación de los cuartiles.

Conviene subrayar que, a pesar de desconocerse la epidemiología local de la enfermedad, por tratarse de una zona tropical andina relativamente cercana al ecuador, y con una altura considerable, una población en su mayoría rural ${ }^{34,35)}$ y una alimentación basada en el maíz, se considera que su baja incidencia puede relacionarse con factores protectores de la enfermedad. Estos serían una mayor radiación ultravioleta y la disponibilidad de ácido linoleico, que intervienen en varios objetivos reconocidos en su fisiopatología, como son la regulación de la migración de células presentadoras de antígeno y el estímulo de la producción de interleucina 2, inhibidor por excelencia de la producción de citocinas 
proinflamatorias $^{(7)}$.

En segundo lugar, el diseño del estudio limitó la posibilidad de seguimiento con la 'durometría' en función del esquema terapéutico, lo cual resultó en la obtención y el análisis de datos puntuales, en una sola consulta clínica.

Otra debilidad detectada fue la ausencia del elemento ciego al momento de registrar los datos, elevando de cierta forma el riesgo de sesgo en las mediciones intraevaluador. Por otro lado, es necesario recalcar que, sabiendo que los promedios de dureza en la piel 'sana' control en su mayoría correspondieron a rangos bajos (<22 unidades de dureza), está bien demostrado que el fenómeno de histéresis en la piel sin manifestaciones clínicas de la enfermedad, hace que difiera significativamente con la biomecánica de la piel de un individuo sin psoriasis ${ }^{(32,33)}$. Esto hace necesaria una muestra de control de individuos sin la enfermedad, para evitar sesgos o errores de medición, otro punto que se debe considerar en estudios futuros.

Ahora bien, lo positivo es que se crea una nueva oportunidad para llevar a cabo más estudios complementarios sobre la 'durometría' en la psoriasis vulgar, con toma de medidas en momentos diferentes, bajo determinado esquema terapéutico, utilizando nuestros parámetros técnicos, todos afinados durante este proceso investigativo, a fin de llegar a una futura validación de su reproducibilidad, sensibilidad y especificidad con una muestra mayor. Se debe tener en cuenta a cada paciente como unidad de análisis, y no simplemente obtener una medida 'fría' de la placa psoriásica con el instrumento, en forma aislada del contexto clínico.

\section{CONCLUSIONES Y RECOMENDACIONES}

Aunque la 'durometría' no se correlaciona con el PASI, es teóricamente factible la recalibración del ítem 'induración' en función del durómetro, para lo cual se requieren más estudios de validación, teniendo en cuenta variables como el esquema terapéutico, las enfermedades asociadas y la calidad de vida de los pacientes con psoriasis vulgar.

Se destaca el uso del durómetro como instrumento objetivo en la valoración puntual de la dureza física de la placa de psoriasis, con excelente correlación y concordancia intraevaluador e interevaluador, tanto para la piel enferma como para la sana.

Se abre una puerta a la 'durometría' en el estudio de la psoriasis en placas, como línea de investigación, bajo unas sencillas premisas teóricas y técnicas que la con- vierten en una herramienta de uso fácil y breve para los dermatólogos expertos o inexpertos.

Como recomendación final, es necesario tener centros de referencia para enfermedades de gran impacto en cada región del país, que nutran las distintas líneas de investigación. En nuestro caso en particular, sería una clínica de psoriasis que nos permita atender una mayor cantidad de pacientes, con valores de PASI en promedio de 11, en los cuales es más probable encontrar placas no localizadas en las prominencias óseas, dando lugar a estudios con resultados de mayor peso e impacto científico.

\section{REFERENCIAS}

1. Boehncke WH. Etiology and pathogenesis of psoriasis. Rheum Dis Clin North Am. 2015;41:665-75.

2. Boehncke WH, Schon MP. Psoriasis. Lancet. 2015;386:983-94.

3. Chalela J, González C, Castro L. Guías de manejo de psoriasis: consenso colombiano. Bogotá: Asociación Colombiana de Dermatología y Cirugía Dermatológica; 2008.

4. González C, Londoño A, Castro L. Guías basadas en la evidencia para el manejo de la psoriasis en Colombia. Bogotá: Editorial Panamericana; 2012. p. 23-67.

5. Kim J, Nadella P, Kim DJ, Brodmerkel C, Correa da Rosa JC, Krueger JG, et al. Histological stratification of thick and thin plaque psoriasis explores molecular phenotypes with clinical implications. PLoS One. 2015;10:e0132454.

6. Naldi L, Gambini D. The clinical spectrum of psoriasis. Clin Dematol. 2007;25:510-8.

7. González C, Castro L, De La Cruz G, Arenas CM, Beltrán A, Santos AM. Caracterización epidemiológica de la psoriasis en el Hospital Militar Central. Rev Asoc Col Dermatol. 2009;17:11-7.

8. Hoffmann K, Dirschka T, Schwarze H, el-Gammal $\mathrm{S}$, Matthes U, Hoffmann A, et al. $20 \mathrm{MHz}$ sonography, colorimetry and image analysis in the evaluation of psoriasis vulgaris. J Dermatol Sci. 1995;9:103-10.

9. Hamilton M, Ntais D, Griffiths C, Davies L. Psoriasis treatment and management - a systematic review of full economic evaluations. Br J Dermatol. 2015;172:574-83.

10. National Clinical Guideline Centre. Psoriasis: Assessment and management of psoriasis. London: Royal College of Physicians; 2012. 
11. PASI Training. A comprehensive training gude to Psoriasis Area Severity index http://www.pasitraining.com/calculator/step_1.php.

12. Alfonso-Trujillo I, Díaz-García MA, RodríguezGarcía MA, Torres-Gemeil O, Falcón-Lincheta L, Pérez-Hernández M, et al. Psoriasis. Glosario para ensayos clínicos. Dermatol Peru. 2007;17:40-3.

13. Kissin EY, Schiller AM, Gelbard RB, Anderson JJ, Falanga V, Simms RW, et al. Durometry for the assessment of skin disease in systemic sclerosis. Arthritis Rheum. 2006;55:603-9.

14. Choh CT, Wall ML, Brown MD, Nicolson AM, Simms MH. Use of durometry in assessment of venous disease. Phlebology. 2010;25:94-9.

15. Falanga V, Bucalo B. Use of a durometer to assess skin hardness. J Am Acad Dermatol. 1993;29:47-51.

16. Mcgraw C, Alexander E Jr. Durometer for measurement of intracranial pressure. Surg Neurol. 1977;7:293-5.

17. Sideris DA, Harocopos FS, Karamitsos CB, Moulopoulos SD. Direct measurement of myocadial hardness. Eur J Cardiol. 1978;7:59-70.

18. Belyaev O, Herden H, Meier JJ, Muller CA, Seelig $\mathrm{MH}$, Herzog $\mathrm{T}$, et al. Assessment of pancreatic hardness -surgeon versus durometer. J Surg Res. 2010;158:53-60.

19. Leblanc N, Falabella A, Murata H, Hasan A, Weiss E, Falanga V. Durometer measurements of skin induration in venous disease. Dermatol Surg. 1997;23:285-7.

20. Seyger MM, van den Hoogen FH, de Boo T, de Jong EM. Reliability of two methods to assess morphea: skin scoring and the use of a durometer. J Am Acad Dermatol. 1997;37:793-6.

21. Poff S, Li SC, Kelsey CE, Foeldvari I, Torok KS. Durometry as an outcome measure in juvenile localized scleroderma. Br J Dermatol. 2016;174:228-30.

22. Merkel PA, Silliman NP, Denton CP, Furst DE, Khanna D, Emery P, et al. Validity, reliability, and feasibility of durometer measurements of scleroderma skin disease in a multicenter treatment trial. Arthritis Rheum. 2008;59:699-705.

23. John OP, Benet-Martínez V. Measurement: Reliability, construct validation, and scale construction. In: Reis HT, Judd CM, editors. Handbook of research methods in social and personality psychology. New York: Cambridge University. Press; 2000. p. 339-69.

24. Aghassi D, Monoson T, Braverman I. Reproducible measurements to quantify cutaneous involvement in scleroderma. Arch Dermatol. 1995;131:1160-6.
25. Naldi L. Scoring and monitoring the severity of psoriasis. What is the preferred method? What is the ideal method? Is PASI passé? Facts and controversies. Clin Dermatol. 2010;28:67-72

26. Purzycka-Bohdan D, Szczerkowska-Dobosz A, Zablotna M, Wierzbicka J, Piotrowska A, Zmijewski MA, et al. Assessment of interleukin 16 serum levels and skin expression in psoriasis patients in correlation with clinical severity of the disease. PLoS One. 2016;11:e0165577.

27. Okan G, Baki AM, Yorulmaz E, Doğru-Abbasoğlu S, Vural P. Fibroblast growth factor 23 and placental growth factor in patients with psoriasis and their relation to disease severity. Ann Clin Lab Sci. 2016;46:174-9.

28. Rutter KJ, Watson RE, Cotterell LF, Brenn T, Griffiths CE, Rhodes LE. Severely photosensitive psoriasis: a phenotypically defined patient subset. J Invest Dermatol. 2009;129:2861-7.

29. Musumeci ML, Lacarrubba F, Fusto CM, Micali G. Combined clinical, capillaroscopy and ultrasound evaluation during treatment of plaque psoriasis with oral cyclosporine. Int J Immunopathol Pharmacol. 2013;26:1027-33.

30. Zafar H, Enfield J, O'Connell ML, Ramsay B, Lynch M, Leahy M. Assessment of psoriatic plaque in vivo with correlation mapping optical coherence tomography. Skin Res Technol. 2014;20:141-6.

31. Shrivastava VK, Londhe ND, Sonawane RS, Suri JS. First review on psoriasis severity risk stratification: An engineering perspective. Comput Biol Med. 2015;63:52-63.

32. Dobrev H. In vivo study of skin mechanical properties in psoriasis vulgaris. Acta Derm Venereol. 2000;80:263-6.

33. Choi JW, Kwon SH, Youn JI, Youn SW. Objective measurements of erythema, elasticity and scale could overcome the inter- and intra-observer variations of subjective evaluations for psoriasis severity. Eur J Dermatol. 2013;23:224-9.

34. Chandran V, Raychaudhuri SP. Geoepidemiology and environmental factors of psoriasis and psoriatic arthritis. J Autoimmun. 2010;34:J314-21.

35. Parisi R, Symmons DP, Griffiths CE, Ashcroft DM; Identification and Management of Psoriasis and Associated ComorbidiTy (IMPACT) project team. Global epidemiology of psoriasis: A systematic review of incidence and prevalence. J Invest Dermatol. 2013;133:377-85. 